\title{
Generalized dressing method for nonlinear evolution equations describing partially coherent wave propagation in noninstantaneous Kerr media
}

\author{
T. Hansson, M. Lisak, and D. Anderson \\ Department of Earth and Space Sciences, Chalmers University of Technology, SE 41296 Göteborg, Sweden
}

(Received 8 July 2011; published 4 November 2011)

\begin{abstract}
A generalized dressing method is presented for integration of the nonlinear evolution equation of the coherent density function describing partially coherent wave propagation in a noninstantaneous Kerr media. As an example an exact, partially coherent multisource $\mathrm{N}$-soliton solution is derived. It is also demonstrated how this method is applicable for construction of solutions to the equivalent coupled system of nonlinear Schrödinger equations of the self-consistent multimode theory.
\end{abstract}

DOI: 10.1103/PhysRevE.84.056601

PACS number(s): 42.65.Tg, 42.65.Sf

\section{INTRODUCTION}

Nonlinear wave propagation is of fundamental interest in a wide range of very diverse areas of physics such as nonlinear optics, plasma physics, and water waves, as well as Bose-Einstein condensates. Generally speaking, most weakly nonlinear, dispersive and/or diffractive, energy-preserving coherent wave systems give rise, in the appropriate limit, to the nonlinear Schrödinger (NLS) equation. The NLS equation provides a "canonical" description of the wave envelope dynamics in a nonlinear medium when dissipation can be neglected, and, specifically, it admits the well-known bright soliton solutions existing due to a stable balance between two counteracting effects, e.g., dispersion or diffraction and self-focusing. It has also been well known for many years that the initial value problem for coherent waves governed by the NLS equation can be fully solved using the inverse scattering transform (IST) method [1].

Until the middle of the 1990s, the commonly held impression was that solitons were inherently coherent structures with well-defined amplitudes and phases [2]. However, experimental results in nonlinear optics have demonstrated that partially coherent solitons are also possible. A necessary condition for the existence of such structures is that the response time of the medium is long as compared to the coherence time of the wave field, so that the medium experiences only the statistical average of the wave field intensity. Partially incoherent solitons have been first found in photorefractive crystals [3], but have rapidly become a concept that is relevant for many different physical areas beyond nonlinear optics [4,5].

Four main formalisms have been developed for theoretical analysis of the propagation of partially coherent waves in nonlinear media: the mutual coherence function approach [6], the self-consistent multimode theory [7], the coherent density theory [8], and the Wigner transform method [9]. These seemingly different formalisms have been shown to be formally equivalent $[10,11]$, and a general mathematical basis unifying the existing approaches has been developed in Ref. [12]. This theory also provides the generalization necessary for analyzing situations where the wave field originates from several sources, which do not necessarily have the same stochastic properties. However, to date, only a few exact solutions have been found in either formalism except for media with a saturable logarithmic nonlinearity [13]. The exception is the self- consistent multimode theory, which is described by a system of incoherently coupled nonlinear Schrödinger equations. Here several analytical solutions have been presented also for Kerr media, although only a few of them satisfy the condition of also being mutually orthogonal [14-17].

Even though the coupled nonlinear Schrödinger system has long been known to be treatable using the IST method [18], it has thus far not been fully realized that the equations governing the propagation of partially coherent light are, in fact, integrable and support an infinite number of conserved quantities [19]. The main purpose of this paper is to present a generalized dressing method for solving the evolution equation of the coherent density approach for partially coherent waves propagating in nonlinear and noninstantaneous Kerr media. The procedure is similar to the Zakharov and Shabat scheme developed in Ref. [20]. The method is applied to obtain a partially coherent multisource $\mathrm{N}$-soliton solution. It is also demonstrated how this method is applicable for construction of solutions to the coupled nonlinear Schrödinger equations of the self-consistent multimode theory. The solution of this system has previously been considered using the dressing method, however, without the additional constraint of orthogonal modal functions; see, e.g., Ref. [21] for a review.

\section{COHERENT DENSITY THEORY}

The coherent density theory was the first formalism developed for describing propagation of partially coherent light in noninstantaneous nonlinear media [22]. The governing equation for the coherent density function $f(x, z, \theta)$ in a Kerr medium is given by

$$
i\left(\frac{\partial f}{\partial z}+\theta \frac{\partial f}{\partial x}\right)+\beta \frac{\partial^{2} f}{\partial x^{2}}+\kappa\left(\int_{-\infty}^{\infty}|f|^{2} d \theta\right) f=0,
$$

where $z$ is the propagation distance, $x$ is transverse coordinate of the beam, $\theta$ represents an angle with respect to the propagation axis, and $\beta$ and $\kappa$ are diffraction and nonlinear coefficients, respectively. Equation (1) may be seen as the continuous limit of an infinite set of coupled nonlinear Schrödinger equations. Indeed, introducing a bra-ket notation 
and interpreting the $\theta$ variable as a continuous index we can apply the transformation

$$
f(x, z, \theta) \exp \left(i \frac{\theta}{2 \beta} x-i \frac{\theta^{2}}{4 \beta} z\right) \rightarrow|\psi(x, z ; \theta)\rangle
$$

to Eq. (1) and obtain

$$
i \frac{\partial}{\partial z}|\psi\rangle+\beta \frac{\partial^{2}}{\partial x^{2}}|\psi\rangle+\kappa\langle\psi \mid \psi\rangle|\psi\rangle=0,
$$

where the ket $|\psi\rangle$ is taken to be an infinite dimensional column vector, and the bra $\langle\psi|$ its Hermitian conjugate row vector. The integral has been replaced by the scalar product $\left\langle\psi_{1} \mid \psi_{2}\right\rangle=$ $\int_{-\infty}^{\infty} \psi_{1}^{*} \psi_{2} d \theta$. Clearly, if $|\psi\rangle$ were an $n$-dimensional vector, Eq. (3) would be equivalent to the following system of coupled nonlinear Schrödinger (CNLS) equations:

$$
i \frac{\partial \psi_{n}}{\partial z}+\beta \frac{\partial^{2} \psi_{n}}{\partial x^{2}}+\kappa\left(\sum_{k=1}^{N}\left|\psi_{k}\right|^{2}\right) \psi_{n}=0, \quad n=1, \ldots, N
$$

and the following analysis is consequently also valid for construction of solutions to a CNLS-system of arbitrary size, given that $|\psi\rangle$ is taken to be a finite dimensional vector. In fact, for mutually orthogonal functions $\psi_{n}$, Eq. (4) is the evolution equation for the modal functions in the selfconsistent multimode theory [23].

\section{ZAKHAROV-SHABAT SCHEME}

Zakharov and Shabat [1] integrated the nonlinear Schrödinger equation in 1971 by means of the inverse scattering transform. Later in 1974 they presented a general scheme for integrating nonlinear evolution equations by means of inverse scattering [20], including a simple technique for constructing particular solutions to nonlinear equations from solutions of the corresponding linear scattering problem, a technique commonly referred to as the dressing method. Below we give a short presentation of the scheme following essentially Refs. [20] and [24].

We start by introducing a linear integral operator $\hat{F}$ such that

$$
\hat{F} \psi(x ; z)=\int_{-\infty}^{\infty} F(x, t ; z) \psi(t ; z) d t .
$$

It is further assumed that this operator can be written in terms of two other integral operators $\hat{K}_{+}$and $\hat{K}_{-}$with kernels satisfying $K_{+}(x, y ; z)=0$ for $y<x$ and $K_{-}(x, y ; z)=0$ for $y>x$, respectively, by means of the relation

$$
I+\hat{F}=\left(I+\hat{K}_{+}\right)^{-1}\left(I+\hat{K}_{-}\right) .
$$

From Eq. (6) it follows that the operator kernels for $y>x$ satisfy the Gel'fand-Levitan integral equation:

$$
F(x, y ; z)+K_{+}(x, y ; z)+\int_{x}^{\infty} K_{+}(x, t ; z) F(t, y ; z) d t=0 .
$$

We now introduce two matrix differential operators depending on a constant parameter $p$, namely,

$$
\Delta_{0}^{(1)}=\tilde{I}\left(i \frac{\partial}{\partial z}+p \frac{\partial^{2}}{\partial x^{2}}\right)
$$

and

$$
\Delta_{0}^{(2)}=\tilde{I}\left[\begin{array}{ll}
l_{1} & 0 \\
0 & l_{2}
\end{array}\right] \frac{\partial}{\partial x},
$$

where $l_{1}=(1-p) / 2 p, l_{2}=(1+p) / 2 p$, and

$$
\tilde{I}=\left[\begin{array}{cc}
|1\rangle\langle 1| & 0 \\
0 & 1
\end{array}\right]
$$

is a generalization of the unit matrix with $|1\rangle$ being the identity ket. The differential operators $\Delta_{0}^{(1)}$ and $\Delta_{0}^{(2)}$ are assumed to satisfy the commutator relations $\left[\Delta_{0}^{(1)}, \hat{F}\right]=0$ and $\left[\Delta_{0}^{(2)}, \hat{F}\right]=$ 0 , which by Eq. (6) implies that we may define so-called dressed operators $\Delta^{(j)}=\left(I+\hat{K}_{-}\right) \Delta_{0}^{(j)}\left(I+\hat{K}_{-}\right)^{-1}$ satisfying

$$
\Delta^{(j)}\left(I+\hat{K}_{+}\right)-\left(I+\hat{K}_{+}\right) \Delta_{0}^{(j)}=0, \quad j=1,2 .
$$

We take the dressed operators to be

$$
\begin{aligned}
\Delta^{(1)} & =\Delta_{0}^{(1)}+2 p \frac{d}{d x} K_{+}(x, x ; z) \\
& =\tilde{I}\left(i \frac{\partial}{\partial z}+p \frac{\partial^{2}}{\partial x^{2}}\right)+2 p\left[\begin{array}{cc}
|\psi\rangle\langle\psi| / l_{1} & \left|\psi_{x}\right\rangle \\
\left\langle\psi_{x}\right| & -\langle\psi \mid \psi\rangle / l_{2}
\end{array}\right]
\end{aligned}
$$

and

$$
\Delta^{(2)}=\tilde{I}\left[\begin{array}{ll}
l_{1} & 0 \\
0 & l_{2}
\end{array}\right] \frac{\partial}{\partial x}+\left[\begin{array}{cc}
0 & -|\psi\rangle \\
\langle\psi| & 0
\end{array}\right] .
$$

The operators satisfy the condition $\left[\Delta^{(1)}, \Delta^{(2)}\right]=0$ or explicitly for $|\psi\rangle$

$$
i \frac{\partial}{\partial z}|\psi\rangle+\frac{\partial^{2}}{\partial x^{2}}|\psi\rangle+\frac{8 p^{2}}{1-p^{2}}\langle\psi \mid \psi\rangle|\psi\rangle=0
$$

and its Hermitian conjugate for $\langle\psi|$. Using the scaling $z \rightarrow$ $z / \beta$ and identifying $\kappa / \beta=2 /\left(l_{1} l_{2}\right)=8 p^{2} /\left(1-p^{2}\right)$, we thus obtain Eq. (3) as a consistency requirement.

\section{DRESSING METHOD}

The dressing method is a general method for nonlinear equations integrable by inverse scattering that allows us to turn a solution for a set of linear equations into a corresponding solution of the full nonlinear problem. More specifically, the dressing method enables us to find solutions of Eq. (14) by constructing a particular solution $F(x, y ; z)$ for the pair of linear partial differential equations $\left[\Delta_{0}^{(1)}, \hat{F}\right]=0$ and $\left[\Delta_{0}^{(2)}, \hat{F}\right]=0$. Using the Gel'fand-Levitan equation (7) we then solve for the kernel $K_{+}(x, y ; z)$, and extract the corresponding solution $|\psi\rangle$; cf. the operator definition (12). As an example we now derive a general partially coherent multisource $\mathrm{N}$-soliton solution.

From $\left[\Delta_{0}^{(1)}, \hat{F}\right]=0$ we get the equation

$$
i \frac{\partial F}{\partial z}+p \frac{\partial^{2} F}{\partial x^{2}}-p \frac{\partial^{2} F}{\partial y^{2}}=0
$$


while $\left[\Delta_{0}^{(2)}, \hat{F}\right]=0$ yields

$$
\left[\begin{array}{ll}
l_{1} & 0 \\
0 & l_{2}
\end{array}\right] \frac{\partial F}{\partial x}+\frac{\partial F}{\partial y}\left[\begin{array}{ll}
l_{1} & 0 \\
0 & l_{2}
\end{array}\right]=0 .
$$

Assuming that

$$
F(x, y ; z)=\left[\begin{array}{cc}
0 & -\left|f_{1}(x, y ; z)\right\rangle \\
-\left\langle f_{2}(x, y ; z)\right| & 0
\end{array}\right],
$$

we see from Eq. (16) that $\left|f_{1}\right\rangle=\left|f_{1}(\xi ; z)\right\rangle$ with $\xi=l_{2} x-l_{1} y$ and $\left\langle f_{2}\right|=\left\langle f_{2}(\eta ; z)\right|$ with $\eta=l_{1} x-l_{2} y$, and Eq. (15) reduces to

$$
\begin{aligned}
& i \frac{\partial}{\partial z}\left|f_{1}\right\rangle+\frac{\partial^{2}}{\partial \xi^{2}}\left|f_{1}\right\rangle=0 \\
& i \frac{\partial}{\partial z}\left\langle f_{2}\right|-\frac{\partial^{2}}{\partial \eta^{2}}\left\langle f_{2}\right|=0
\end{aligned}
$$

since $p\left(l_{2}^{2}-l_{1}^{2}\right)=1$.

We further assume that the $\theta$ dependence enters only in the phase, in which case solutions of the system Eqs. (18)-(19) corresponding to solitons with distinct eigenvalues take the form

$$
\begin{gathered}
\left|f_{1}\right\rangle=\sum_{n=1}^{N}\left|r_{n}(\theta)\right\rangle \exp \left[\rho_{n} \xi+i \rho_{n}^{2} z\right], \\
\left\langle f_{2}\right|=\sum_{n=1}^{N}\left\langle r_{n}(\theta)\right| \exp \left[-\rho_{n}^{*} \eta-i \rho_{n}^{* 2} z\right],
\end{gathered}
$$

where $\left|r_{n}(\theta)\right\rangle$ are complex functions whose real and imaginary parts are related to the soliton position and phase, respectively, while $\rho_{n}=v_{n}+i \mu_{n}$ are complex constants with $v_{n}>0$ being the soliton amplitude (width) and $\mu_{n}$ its transverse velocity. Introducing

$$
K_{+}(x, y ; z)=\left[\begin{array}{ll}
a(x, y ; z) & b(x, y ; z) \\
c(x, y ; z) & d(x, y ; z)
\end{array}\right]
$$

we have $|\psi\rangle=b(x, x ; z)$ according to Eq. (12), and from Eq. (7) we obtain an integral equation for $b(x, y ; z)$, namely,

$b(x, y ; z)=\left|f_{1}(x, y ; z)\right\rangle+\int_{x}^{\infty} b(x, t ; z) \Gamma(t, x, y ; z) d t$,

where

$$
\begin{aligned}
\Gamma(t, x, y ; z)= & \int_{x}^{\infty}\left\langle f_{2}\left(t, t^{\prime} ; z\right) \mid f_{1}\left(t^{\prime}, y ; z\right)\right\rangle d t^{\prime} \\
= & -\sum_{j, n=1}^{N} \frac{\left\langle r_{j} \mid r_{n}\right\rangle}{l_{2}\left(\rho_{n}+\rho_{j}^{*}\right)} \exp \left[l_{2}\left(\rho_{n}+\rho_{j}^{*}\right) x\right. \\
& \left.-l_{1} \rho_{j}^{*} t-l_{1} \rho_{n} y+i\left(\rho_{n}^{2}-\rho_{j}^{* 2}\right) z\right] .
\end{aligned}
$$

Assuming $b(x, y ; z)=\sum_{k=1}^{N} \tilde{B}_{k}(x ; z) \exp \left(-l_{1} \rho_{k} y\right)$, we match the parts having the same $y$ dependence in Eq. (23) to obtain a coupled system of linear algebraic equations for $\tilde{B}_{n}$, which is used to find the desired solution of Eq. (14). Thus we have the system

$$
B_{n}+\sum_{j, k=1}^{N} \alpha_{n k j} B_{k}=\gamma_{n}, \quad n=1, \ldots, N,
$$

where we have defined $B_{n}=\tilde{B}_{n} \exp \left(-l_{1} \rho_{n} x\right), \gamma_{n}=\left|r_{n}(\theta)\right\rangle$ $\exp \left[\rho_{n} x+i \rho_{n}^{2} z\right]$ and

$$
\begin{aligned}
\alpha_{n k j}= & \frac{\left\langle r_{j} \mid r_{n}\right\rangle}{l_{1} l_{2}\left(\rho_{n}+\rho_{j}^{*}\right)\left(\rho_{k}+\rho_{j}^{*}\right)} \\
& \times \exp \left[\left(\rho_{n}+\rho_{j}^{*}\right) x+i\left(\rho_{n}^{2}-\rho_{j}^{* 2}\right) z\right] .
\end{aligned}
$$

The solution $|\psi\rangle$ is explicitly obtained through Cramer's rule as

$$
\begin{aligned}
|\psi(x, z, \theta)\rangle & =\sum_{n=1}^{N} B_{n}(x ; z)=\sum_{n=1}^{N} \frac{\operatorname{det}\left[\mathbf{a}_{1} \ldots \mathbf{a}_{n-1} \gamma \mathbf{a}_{n+1} \ldots \mathbf{a}_{N}\right]}{\operatorname{det}(\mathbf{A})} \\
& =\frac{\operatorname{det}\left[\gamma \mathbf{a}_{2}-\mathbf{a}_{1} \mathbf{a}_{3}-\mathbf{a}_{1} \ldots \mathbf{a}_{N}-\mathbf{a}_{1}\right]}{\operatorname{det}(\mathbf{A})}
\end{aligned}
$$

where $\mathbf{a}_{k}$ denotes column $k$ of the coefficient matrix $\mathbf{A}=$ $\delta_{n k}+\sum_{j} \alpha_{n k j}$ of Eq. (25).

\section{SOLITON SOLUTIONS}

Equation (27) is valid both as a solution for the infinite dimensional coherent density formalism and for the finite dimensional CNLS-system (4). It can be seen as describing multisource soliton solutions with mutual correlations determined by the factors $\left\langle r_{j} \mid r_{n}\right\rangle$. For uncorrelated sources the CNLS solutions can directly be taken as modal functions in the self-consistent multimode theory. In what follows, we consider the more general coherent density solutions.

The single soliton solution of Eq. (14) is obtain from Eqs. (25)-(27) for $N=1$. Setting $\rho_{1}=v+i \mu$ and $\left|r_{1}\right\rangle=$ $2 \nu \sqrt{J(\theta) l_{1} l_{2}} \exp \left(-v x_{0}+i \varphi\right)$, where $J(\theta)$ is the normalized angular power spectrum of the source, and $\int J(\theta) d \theta=1$, we find

$$
\begin{aligned}
|\psi(x, z, \theta)\rangle= & v \sqrt{J(\theta) l_{1} l_{2}} \operatorname{sech}\left[v\left(x-x_{0}-2 \mu z\right)\right] \\
& \times \exp \left[i \mu x+i\left(v^{2}-\mu^{2}\right) z+i \varphi\right],
\end{aligned}
$$

which is a solution of the Manakov type; cf. Ref. [18]. It reduces to the coherent single-soliton solution in the limit $J(\theta) \rightarrow \delta(\theta)$. Similarly, one can construct single-source Nsoliton solutions by taking $\left|r_{n}\right\rangle=\sqrt{J(\theta)} C_{n}$, where $C_{n}$ are arbitrary complex constants.

To illustrate the construction of a partially coherent solution we consider the case of two partially correlated sources. The coherent density function may then be expressed as a sum of two spatial modulation functions weighted by the angular power spectrum of each source, viz.,

$$
\begin{aligned}
|\psi(x, z, \theta)\rangle & =\left|\psi_{1}(x, z, \theta)\right\rangle+\left|\psi_{2}(x, z, \theta)\right\rangle \\
& =\sqrt{J_{1}(\theta)} W_{1}(x, z)+\sqrt{J_{2}(\theta)} W_{2}(x, z),
\end{aligned}
$$

where the modulation functions $W_{n}(x, z)$ are directly obtained by means of a cofactor expansion along the first column of the determinant in the numerator of the last expression of Eq. (27). The amount of mutual correlation between the sources is characterized by the correlation coefficient $R=$ $\int \sqrt{J_{1}^{*}(\theta) J_{2}(\theta)} d \theta$, where $|R| \leqslant 1$ when the angular power spectra are normalized as $\int J_{n}(\theta) d \theta=1$. 
It follows from Eqs. (25)-(27) with $N=2$ that the twosource problem has a general solution of the form

$$
\begin{aligned}
& |\psi(x, z, \theta)\rangle \\
& =\frac{\left|r_{1}\right\rangle}{\operatorname{det}(\mathbf{A})} \exp \left(\rho_{1} x+i \rho_{1}^{2} z\right) \\
& \quad \times\left[\left(1+\alpha_{221}+\alpha_{222}\right)-\left(\alpha_{211}+\alpha_{212}\right)\right] \\
& \quad+\frac{\left|r_{2}\right\rangle}{\operatorname{det}(\mathbf{A})} \exp \left(\rho_{2} x+i \rho_{2}^{2} z\right) \\
& \quad \times\left[\left(1+\alpha_{111}+\alpha_{112}\right)-\left(\alpha_{121}+\alpha_{122}\right)\right],
\end{aligned}
$$

where $\alpha_{n k j}$ is defined as in Eq. (26) and

$$
\begin{aligned}
\operatorname{det}(\mathbf{A})= & \left(1+\alpha_{111}+\alpha_{112}\right)\left(1+\alpha_{221}+\alpha_{222}\right) \\
& -\left(\alpha_{121}+\alpha_{122}\right)\left(\alpha_{211}+\alpha_{212}\right) .
\end{aligned}
$$

The solution (30) is valid for sources with arbitrary angular power spectra and will generally by periodic in $z$ for vanishing soliton velocities. However, contrary to the single-source twosoliton case, there is also a stationary case obtained when the two sources are uncorrelated with each other and the solitons' velocities $\mu_{n}$ are chosen to be zero. This implies from Eq. (26) that $\alpha_{n k j}=\alpha_{n k j} \delta_{n j}$ and Eqs. (30)-(31) reduce to

$$
\begin{aligned}
|\psi(x, z, \theta)\rangle= & \sqrt{J_{1}(\theta) l_{1} l_{2}\left(v_{1}^{2}-v_{2}^{2}\right)} \frac{v_{1} \operatorname{csch}\left[v_{1}\left(x-x_{1}\right)\right]}{v_{1} \operatorname{coth}\left[v_{1}\left(x-x_{1}\right)\right]-v_{2} \tanh \left[v_{2}\left(x-x_{2}\right)\right]} \exp \left(i v_{1}^{2} z+i \varphi_{1}\right) \\
& +\sqrt{J_{2}(\theta) l_{1} l_{2}\left(v_{1}^{2}-v_{2}^{2}\right)} \frac{v_{2} \operatorname{sech}\left[v_{2}\left(x-x_{2}\right)\right]}{v_{1} \operatorname{coth}\left[v_{1}\left(x-x_{1}\right)\right]-v_{2} \tanh \left[v_{2}\left(x-x_{2}\right)\right]} \exp \left(i v_{2}^{2} z+i \varphi_{2}\right),
\end{aligned}
$$

where we have assumed that $v_{1}>v_{2}$. In fact, for uncorrelated sources we see that by dropping the multiplicative $\theta$ dependencies each field $\left|\psi_{n}\right\rangle$ is a multimode solution and satisfies a CNLS equation (4). For the special case with $v_{1}=2 v_{2}$ and $x_{1}=x_{2}=0$, Eq. (32) reduces to a solution with a sech-squared intensity profile previously found for the CNLS equation in Ref. [15].

When the two sources are neither completely correlated as in the coherent two-soliton case nor completely uncorrelated, i.e., when $0<|R|<1$, we can still write the solution Eq. (30) in a canonical form as the sum of two mutually orthogonal fields $|\psi\rangle=\left|\tilde{\psi}_{1}\right\rangle+\left|\tilde{\psi}_{2}\right\rangle$ with $\left\langle\tilde{\psi}_{1} \mid \tilde{\psi}_{2}\right\rangle=0$. This is accomplished by diagonalizing the correlation matrix $\left\langle\psi_{j} \mid \psi_{n}\right\rangle$, a procedure, which we have described in detail in our previous paper [12]. Thus the corresponding multimode solution can be found from the concomitant coherent density solution. The canonical form is also helpful when considering interactions between solitons; cf. Ref. [17].

\section{CONCLUSIONS}

The evolution equations governing partially coherent waves propagating in noninstantaneous Kerr media have been shown to be integrable by the method of inverse scattering. However, the full machinery of the inverse scattering method is often cumbersome to apply. In this paper we have therefore demonstrated how the simpler dressing method can be used for the construction of partially coherent soliton solutions for the coherent density formalism and the self-consistent multimode theory. As an example, we derived an exact partially coherent multisource N-soliton solution. This solution represents a generalization of a coherent $\mathrm{N}$-soliton solution generated by $\mathrm{N}$ fully correlated sources to one generated instead by sources with arbitrary mutual correlations. We have also discussed how the orthogonal modal functions of the equivalent selfconsistent multimode theory could be obtained from the more general coherent density solution not only for uncorrelated sources but also for sources with mutual correlations.
[1] V. E. Zakharov and A. B. Shabat, Sov. Phys. JETP 34, 62 (1972).

[2] Yu. S. Kivshar and G. P. Agraval, Optical Solitons: From Fibers to Photonic Crystals (Academic, San Diego, 2003).

[3] M. Mitchell, Z. Chen, M. F. Shih, and M. Segev, Phys. Rev. Lett. 77, 490 (1996).

[4] R. Fedele, P. K. Shukla, M. Onorato, D. Anderson, and M. Lisak, Phys. Lett. A 303, 61 (2002).

[5] P. Johannisson, D. Anderson, M. Lisak, M. Marklund, R. Fedele, and A. Kim, Phys. Rev. E 69, 066501 (2004).

[6] G. A. Pasmanik, Sov. Phys. JETP 39, 234 (1974).

[7] M. Mitchell, M. Segev, T. H. Coskun, and D. N. Christodoulides, Phys. Rev. Lett. 79, 4990 (1997).

[8] D. N. Christodoulides, T. H. Coskun, M. Mitchell, and M. Segev, Phys. Rev. Lett. 78, 646 (1997).

[9] B. Hall, M. Lisak, D. Anderson, R. Fedele, and V. E. Semenov, Phys. Rev. E 65, 035602 (2002).
[10] D. N. Christodoulides, E. D. Eugenieva, T. H. Coskun, M. Segev, and M. Mitchell, Phys. Rev. E 63, 035601 (2001).

[11] M. Lisak, L. Helczynski, and D. Anderson, Opt. Commun. 220, 321 (2003).

[12] V. Semenov, M. Lisak, D. Anderson, T. Hansson, L. Helczynski-Wolf, and U. Österberg, J. Phys. A 41, 335207 (2008).

[13] D. N. Christodoulides, T. H. Coskun, M. Mitchell, and M. Segev, Phys. Rev. Lett. 80, 2310 (1998).

[14] N. Akhmediev, W. Krolikowski, and A. W. Snyder, Phys. Rev. Lett. 81, 4632 (1998).

[15] M. I. Carvalho, T. H. Coskun, D. N. Christodoulides, M. Mitchell, and M. Segev, Phys. Rev. E 59, 1193 (1999).

[16] T. Kanna and M. Lakshmanan, Phys. Rev. Lett. 86, 5043 (2001). 
[17] D. Anderson, L. Helczynski-Wolf, M. Lisak, and V. E. Semenov, Opt. Commun. 281, 3919 (2008).

[18] S. V. Manakov, Zh. Eksp. Teor. Fiz. 65, 505 (1973).

[19] T. Hansson, M. Lisak, and D. Anderson (unpublished).

[20] V. E. Zakharov and A. B. Shabat, Funct. Anal. Appl. 8, 226 (1974).

[21] H. Blas, e-print arXiv:solv-int/9912015v1.
[22] D. N. Christodoulides, T. H. Coskun, M. Mitchell, and M. Segev, Phys. Rev. Lett. 78, 646 (1997).

[23] M. Mitchell, M. Segev, T. H. Coskun, and D. N. Christodoulides, Phys. Rev. Lett. 79, 4990 (1997).

[24] P. G. Drazin and R. S. Johnson, Solitons: An Introduction, 2nd ed. (Cambridge University Press, Cambridge, 1989). 